\title{
TRIM59 is Suppressed by Androgen Receptor and Acts to Promote Lineage Plasticity and Neuroendocrine Differentiation in Prostate Cancer
}

\author{
Liancheng Fan \\ Shanghai Jiao Tong University School of Medicine Affiliated Renji Hospital \\ Yiming Gong \\ Shanghai Jiao Tong University School of Medicine Affiliated Renji Hospital \\ Yuman He \\ Shanghai Jiao Tong University School of Medicine Affiliated Renji Hospital \\ Wei-Qiang Gao \\ Shanghai Jiao Tong University School of Medicine Affiliated Renji Hospital

\section{Baijun Dong} \\ Shanghai Jiao Tong University School of Medicine Affiliated Renji Hospital \\ Helen He Zhu \\ Shanghai Jiao Tong University School of Medicine Affiliated Renji Hospital \\ Wei Xue ( $\sim$ 18955035798@163.com ) \\ Shanghai Jiao Tong University School of Medicine Affiliated Renji Hospital
}

Research

Keywords: neuroendocrine differentiation, prostate cancer, lineage plasticity, TRIM59

Posted Date: May 7th, 2021

DOI: https://doi.org/10.21203/rs.3.rs-470260/v1

License: (c) (i) This work is licensed under a Creative Commons Attribution 4.0 International License. Read Full License 


\section{Abstract}

Background: The incidence of treatment-induced neuroendocrine prostate cancer (t-NEPC) has been greatly increasing after the usage of second-generation androgen receptor (AR) pathway inhibitors (ARPIs). Neuroendocrine differentiation (NED) is closely associated with ARPI treatment failure and poor prognosis in prostate cancer (PCa) patients. However, the molecular mechanisms of NED are not fully understood.

Methods: TRIM59 expression was evaluated in PCa samples from patients at first diagnosis or at relapse stage post ARPI treatment by immunohistochemistry; in vitro effects of TRIM59 were determined by cell proliferation, sphere formation and cell migration assays; while in vivo analysis was performed using subcutaneous tumor model. Western blot, qPCR assay, dual luciferase assessment, chromatin immunoprecipitation and RNA sequencing were applied for mechanistic exploration.

Results: Here we report that upregulation of TRIM59, a TRIM family protein, is strongly correlated with ARPI treatment mediated NED and shorter patient survival in PCas. AR binds to TRIM59 promoter and represses its transcription. ARPI treatment leads to a reversal of repressive epigenetic modifications on TRIM59 gene and the transcriptional restraint on TRIM59 by AR. Upregulated TRIM59 then drives the NED of PCa by enhancing the degradation of RB1 and P53 and upregulating downstream lineage plasticity-promoting transcription factor SOX2.

Conclusion: Altogether, TRIM59 is negatively regulated by AR and acts as a key driver for NED in PCas. Our study provides a novel prognostic marker for PCas and shed new light on the molecular pathogenesis of t-NEPC, a deadly variant of PCa.

\section{Background}

Androgen receptor (AR) pathway inhibitors (ARPIs), such as abiraterone, enzalutamide, etc, have become the first-line treatment of metastatic prostate cancers (PCas) $[1,2]$. However, with the application of ARPIs, especially the second-generation ARPIs, the prevalence of a highly aggressive castration resistant prostate cancer (CRPC) subtype named neuroendocrine PCa (NEPC) with small cell carcinoma-like morphology, including indistinct cell borders, high nuclei-cytoplasm ratio, hyperchromatic nuclei and elevated neuroendocrine-related biomarker expression, has been steadily increasing. NEPC now accounts for approximately $20 \%$ in PCa patients who develop resistance to ARPIs, which stands in great contrast to only $1 \%$ in untreated newly diagnosed PCa patients [3]. NEPC patients are now commonly treated with platinum-based chemotherapy, but the disease prognosis is highly unfavorable[4]. Our incomplete understanding of the molecular drivers and regulators in neuroendocrine differentiation (NED) hinders the development of better treatment approaches.

Most of PCas at first diagnosis are histologically adenocarcinoma with a luminal-cell phenotype[5, 6]. Although the mechanisms are still elusive, it is suggested that lineage plasticity and luminal-to-neuroendocrine transdifferentiation contribute to the emergence of treatmentinduced NEPC[7, 8]. Several signaling molecules have been shown to participate in this process. These include concurrent loss or mutation of $R B 1$ and TP53[3, 9-11], increased lineage plasticity associated with the reprogramming factor SOX2[12], upregulation of neuronal transcription factor SOX11[13], BRN2[14], oncogene AURKA[15] and MYCM16-20], splicing factor SRRM4[21], and epigenetic modifier EZH2[18] and etc. It is believed that the use of highly potent ARPIs and the loss of AR signaling are initial triggers of NED $[3,9]$. However,how AR activity loss modulates the molecular events leading to NED remains to be further explored.

TRIM59, a member of the tripartite motif (TRIM)-containing protein family, is comprised of one Ring finger region, one B-box region, two crimped helices and one transmembrane domain[22]. Due to its Ring finger domain, TRIM59 is mainly considered to be a putative E3 ubiquitin ligase [22, 23]. Our previous study has shown that TRIM59 played an oncogenic role in gastric carcinogenesis [24]. Moreover, TRIM59 upregulation has been shown to be a driver of prostatic tumorigenesis in a genetically engineered murine model, in which Trim59 overexpression was controlled by a prostate specific PSP94 gene promoter[25]. Intriguingly, the prostate tumor tissue from PSP94-Trim59 mice displayed features of NEPC and expressed the neuroendocrine marker gene Chga[25]. Therefore, TRIM59 may act as an essential gene in PCa progression and NED. However, the role and molecular mechanism of TRIM59 in castration resistance of human PCa and NED has not been elucidated.

Here we find a prominent elevation of TRIM59 protein levels in human PCa samples after the ARPI treatment. TRIM59 upregulation is strongly correlated with NED in PCas. We further demonstrate that TRIM59 is transcriptionally repressed by AR. TRIM59 promotes degradation of RB1 and P53 and then upregulates downstream SOX2, therefore potentiates the lineage plasticity and drives the NED of PCa.

\section{Methods}

\section{Cell lines}

Cell line LNCaP, LAPC4, 293T, PC3 and DU145, were obtained from ATCC. LNCaP and LNCaP-derived cells were maintained in RMPI-1640 (Gibco) with 10\% FBS (Gibco), containing 80U/ml penicillin and streptomycin. The other cell lines were maintained in DMEM (Gibco) with 10\% 
FBS (Gibco), containing $80 \mathrm{U} / \mathrm{ml}$ penicillin and streptomycin. Cells were cultured in $5 \% \mathrm{CO} 2$ incubators at $37^{\circ} \mathrm{C}$. For androgen deprivation or stimulation, LNCaP cells were incubated with 2nM R1881 (Sigma-Aldrich) with or without 20 $\mu$ M enzalutamide (MCE) 48hrs before subsequent experiments. Cells incubated with vehicle were taken as the control.

\section{Patients}

Patient sample and medical information collection protocols used in this study is approved by the Medical Ethical Committee at Ren Ji Hospital, School of Medicine, Shanghai Jiao Tong University. Patients diagnosed with NEPC $(n=21)$ or CRPC-adenocarcinoma $(n=39)$ from January, 2010 to December, 2019 were enrolled in the NEPC/CRPC-adenocarcinoma cohort. For pathological classification, fresh samples were collected and paraffin embedded. $5 \mu \mathrm{m}$ thickness sections were prepared for H\&E staining to observe cell morphology. When characteristic NEPC morphology as previously described in the manuscript was identified, immunohistochemical staining was further carried out to detect the neuroendocrine markers expression. The diagnosis of NEPC was based on both tumor morphology and elevated neuroendocrine markers by certified pathologists in Department of Pathology, Ren Ji Hospital. In the Renji t-NEPC cohort, 10 androgendependent PCas at the first diagnosis eventually develop into NEPCs after a series of ARPI treatment. Paraffin slices from 10 pairs of sequential pre- and post-ARPI treatment samples were stained for AR, TRIM59, CgA and SYP. All the other samples were stained for TRIM59. IHC were conducted as described previously [26]. Antibodies used for IHC were listed as follows: TRIM59 (ab166793, Abcam), AR (5153, Cell Signaling Technologies), SYP (36406, Cell Signaling Technologies), CgA (ab15160, Abcam).

\section{In vitro sphere forming and migration assay}

The single cell suspension at $10^{4}$ cells/ml were seeded in 6-well low-attachment plates with DMEM-F12 containing $10 \mu M$ enzalutamide. Spheroid $(>100 \mu \mathrm{m})$ were numerated under a light microscope (Zeiss).

For cell migration assay, $2 \times 10^{4}$ cells were plated in the upper chamber with serum-free medium (DMEM for PC3 and DU145, RPMI-1640 for $\mathrm{LNCaP}$ ), separated from FBS-containing medium in the lower chamber. After 24 -hour incubation at $37^{\circ} \mathrm{C}$ in $5 \% \mathrm{CO} 2$, non-migrated cells were removed. The number of migrated cells were stained with crystal violet (Beyotime, C0121) and then counted using microscope in 5 randomly chosen fields.

\section{RNA-seq and RT-PCR}

Total RNAs were extracted from cells using TRIZOL following manufacturer's instruction. Then mRNA was reverse-transcribed to barcoded cDNA fragments using oligo-dT primer with adapter attached. Barcoded cDNA libraries were sequenced using the Illumina HiSeq 4000 PE150 platform. RNA-seq reads were aligned to the reference genome (GRCh37/hg19) using HISAT2 after quality assessment. StringTie was used to assemble and quantitate the abundance of transcripts. DESeq2 was used to perform differential gene expression analysis to the normalized data.

For RT-PCR, reverse transcription was performed using HiScript III RT SuperMix for qPCR (R323-01, Vazyme) according to the manufacturer's instructions. Real-time PCR was conducted in quadruplicates using ChamQ Universal SYBR qPCR Master Mix (Q711-02, Vazyme). Primers used in this study was in the supplementary Table 3.

\section{Immunoblotting}

Whole cell lysates were prepared in Cell lysis Buffer (C500035, Sangon). About $10^{7}$ cells were lysed with $1 \mathrm{ml}$ lysis buffer containing protease inhibitor cocktail (MCE, HY-K0010). SDS-PAGE and immunoblotting experiment was conducted following conventional methods. Antibodies used in immunoblotting included GAPDH (D16H11, Cell Signaling Technologies), TRIM59 (ab166793, abcam), P53 (2527, Cell Signaling Technologies), RB1 (9309, Cell Signaling Technologies), AR (5153, Cell Signaling Technologies), KLK3(YD120801, Epitomics), SYP (36406, Cell Signaling Technologies), CgA (ab15160, abcam), SOX2 (3579, Cell Signaling Technologies). BIO-RAD GelDoc XR was used to detect the blot bands.

\section{Chromatin Immunoprecipitation Assay}

LNCaP cells was treated with 2nM R1881 alone, 2nMR1881 together with 20 $\mu$ M enzalutamide or DMSO vehicle 24hrs before the ChIP assay. ChIP was performed using the SimpleChIP Enzymatic Chromatin IP Kit (Magnetic Beads) (\#9003, Cell Signaling Technologies) according to manufacturer's instructions. In brief, about $2 \times 10^{7}$ cells for each immunoprecipitation were crosslinked for $10 \mathrm{~min}$ in $1 \%$ formaldehyde at roomtemperature. Chromatin was then fragmented by nuclease digestion and sonication. Chromatin Immunoprecipitation was conducted with the following antibodies: rabbit anti-AR antibodies (5153, Cell Signaling Technologies), rabbit anti-H3K4me3 (9751, Cell Signaling Technologies), rabbit anti-H3K27ac (8173, Cell Signaling Technologies), rabbit anti-H3K27me3 (9377, Cell Signaling Technologies), rabbit anti-H3K9me2/3 (5327, Cell Signaling Technologies), normal rabbit IgG (2729, Cell Signaling Technologies) and rabbit anti-histone H3 antibodies (4620, Cell 
Signaling Technologies). Immunoprecipitated DNA was quantified by RT-PCR as previously described with primers designed according to predicted androgen response elements.

\section{Plasmids}

TRIM59 overexpression vector pCMV-TRIM59-3X Flag-GFP, AR overexpression vector and pLK0.1-shAR vector were described in our previous study [27]. shTRIM59-1 and shTRIM59-2 lentiviral plasmids were generously provided by Dr. Haizhong Feng [28]. The other pGIPZ shRNA vectors (shSOX2) were obtained from the plasmid library at School of Medicine, Shanghai Jiao Tong University. shSox2 sequence is "TGCTGTTGACAGTGAGCGCCCATTGTTTATAAGCTGAGAATAGTGAAGCCACAGATGTATTCTCAGCTTATAAACAATGGATGCCTACTGCCTCGGA" and

"TGCTGTTGACAGTGAGCGCAGCGACGAAAAAAATGTTTTATAGTGAAGCCACAGATGTATAAAACATTTTTTTCGTCGCTTTGCCTACTGCCTCGGA". shRB1 sequence is "GATCCGGAAAGGACATGTGAACTTATCTCGAGATAAGTTCACATGTCCTTTCCTTTTTT". shEHMT1 sequence is "cgAGTCAATAACGCCAGCTAT" and "ccTCTTTGATCTCGACAATAA". shEHMT2 sequence is "cgAGAGAGTTCATGGCTCTTT" and "TGCGTGCTGTTATTCCTGTCA". pGL4.17-TRIM59-FL-luciferase plasmid was constructed by insertion of a kpn I/Xhol fragment containing TRIM59 promoter (-1462bp +1032bp) cloned from LNCaP cells together with luciferase CDS in pGL4.17 vector. AR luciferase reporter plasmid was purchased from the Genomeditech company, China (GM-021036). The ARE sequence is

TTGATATGGAGGAACTATTTATTTGGAGGAACATATTGTATTTATT.

\section{Lentiviral transfection}

The lentiviral particle was produced in HEK293T cells after co-transfection with lentiviral plasmid and packaging vectors (psPAX2 and PMD2.G) at a 6:1:7 ratio using Polyetherimide in 10-cm dishes. The lentivirus was harvested 36-60 hours later after replacement of media at 12 hours post-transfection. To generate stable transgenic lines, LNCaP and LAPC4 were infected at a 50\% confluency with the lentiviral particle and $8 \mathrm{ug} / \mathrm{ml}$ polybrene (Sigma-Aldrich). The positively transfected cells were selected and enriched by applying puromycin (5 $\mathrm{gg} / \mathrm{ml})$ in the culture medium for 2 weeks.

\section{Luciferase assay}

TRIM59-promoter-luciferase lentivirus were produced and used for transfecting LNCaP cells. Stably transfected LNCaP cells were seeded in 12-well plate and treated with vehicle (DMSO), 2nM R1881 or 20 $\mu$ M Enzalutamide. Dual-luciferase Reporter Assay System (Promega, E2920) was used to detect firefly luciferase activity $24 \mathrm{hrs}$ post treatment according to the manufacturer's protocol. Firefly luciferase activity was normalized to cell numbers.

\section{Tumor xenograft experiment}

All the mice were kept in the pathogen-free facility at Ren Ji Hospital. $5 \times 10^{6}$ cells were suspended in $100 \mu \mathrm{l} 50 \% \mathrm{Matrigel}$ and injected into the right flank of nude mice. When testing the capacity of in-vivo castration resistance, nude mice were castrated 2 weeks prior to subcutaneous tumor cell implantation. Tumor were harvested, imaged and weighed after sacrifice of the experimental mice.

\section{Protein stability assay}

LNCaP/AR or 293T cells were used for performed protein stablility assays as preciously described[24]. For dose dependent protein stability assay, LNCaP cells was transfected with $1 \mu \mathrm{g}, 5 \mu \mathrm{g}, 10 \mu \mathrm{g}$ TRIM59-Flag or empty vector. All the samples were lysed to detect RB1 and P53 protein levels at 48 hours after transfection. For time dependent protein stability assay, $10^{6} 293 \mathrm{~T}$ cells were co-transfected with $2 \mu \mathrm{g}$ TRIM59Flag vector and $2 \mu \mathrm{g}$ P53 or RB1 expression vector, while $10^{6}$ LNCaP cells was transfected with $5 \mu$ g TRIM59-Flag or empty vector. The samples were lysed at 24 hours, 48 hours and 60 hours after transfection. For MG132 treatment, LNCaP transfected with 10 4 g TRIM59-Flag or empty vector were cultured in medium with $10 \mu \mathrm{g} / \mathrm{ml}$ cycloheximide (Sigma-Aldrich) at 36 hours post-transfection.

\section{Statistical analysis}

All statistical analysis was performed using the GraphPad 7.0 software. Statistical analysis method used in this study was provided in corresponding figure legend. $P<0.05$ is considered significant.

\section{Results}

\section{TRIM59 is highly expressed in castration resistant NEPCs}

To determine the significance of TRIM59 in NEPC, we first analyzed a published NEPC-enriched CRPC dataset (the Beltran-2016 cohort)[9] containing 15 castration resistant NEPC samples and 34 CRPC with adenocarcinoma phenotype (CRPC-AD) samples (Fig. 1A). The NEPCs showed significantly higher mRNA levels of TRIM59 compared to the CRPC-AD subgroup (Fig. 1A, 1B). In addition, the high expression level of 
TRIM59 was significantly associated with worse survival in PCa patients based on data analysis of The Human Protein Atlas prostate cancer dataset (Fig. 1C). To further examine the TRIM59 protein expression in clinical samples, we examined our in-house cohort (the Renji cohort, $n$ $=60$ ) of CRPC specimens including 39 CRPCs with adenocarcinoma phenotype (CRPC-AD) and 21 NEPCs (Fig. 1D). Statistical analysis of the IHC staining result showed that TRIM59 was significantly upregulated in NEPCs than CRPC-AD (Fig. 1E). Consistent with the previous results in The Human Protein Atlas prostate cancer dataset (Fig. 1C), we observed a significantly shorter overall survival (Fig. 1F) and faster progression to abiraterone resistance in high TRIM59 expression group than the low expression group in our cohort (Fig. 1G). Interestingly, in addition to the elevated TRIM59 expression in NEPC, we also found a significant increase of the TRIM59 mRNA level in small cell lung cancers, which share pathohistological similarities with NEPCs, from data analysis at The Cancer Genome Atlas (TCGA) and the SCLC cohort (supplementary Fig. 1A-1C)[29]. Those data suggested a strong association of TRIM59 with neuroendocrine cancers.

\section{TRIM59 is negatively correlated with the AR signaling in human CRPCs}

Since NED often occurs after potent ARPI treatment, we focused on the relationship between TRIM59 and AR. We first analyzed the AR and TRIM59 mRNA level in the Beltran-2016 dataset and found an inverse correlation between AR and TRIM59 transcripts (Fig. 2A, 2B).

Furthermore, we uncovered a significantly negative correlation between expression of AR signature genes (referred as AR score here) in the Beltran-2016 cohort (Fig. 2C). In another published PCa RNA-seq dataset (TCGA firehose), the TRIM59 transcript was also negatively correlated with the expression level of classic AR downstream genes including KLK3, KLK2 and PART1 (supplementary Fig. 2). To further confirm this finding, we performed IHC analysis of TRIM59 and AR on human CRPC samples $(n=44)$. In concordance with the Beltran-2016 dataset analysis, our IHC experiments revealed that AR-positive CRPC samples exhibited low or negative staining for TRIM59, while ARnegative CRPC samples showed high or medium TRIM59 staining (Fig. 2D, 2E). In addition, we assessed TRIM59, AR and SYP protein levels by IHC staining in PCa samples from 10 treatment-induced NEPC (t-NEPC) patients, all of which included hormone-naïve PCa samples before ARPI treatment and t-NEPC samples after ARPI treatment from the same patient. Among them, 8 (80\%) patients showed a marked upregulation of TRIM59 expression when the disease progressed to t-NEPC after ARPI treatment compared to treatment-naive primary PCa (Fig. 2G, 2H). One patient showed no change and one patient sample exhibited a slight decline of TRIM59 expression along the t-NEPC development. Collectively, our findings suggested a robust increase in the TRIM59 expression upon prolonged ARPI treatment in PCa patients.

\section{AR signaling suppresses the transcription of TRIM59 in prostate cancer cells}

We further analyzed a published dataset (GSE71797), wherein LNCaP and VCaP cells were stimulated with R1881. Consistently, we found a reduced expression of TRIM59 when AR was activated by R1881 (Fig. 3A, 3B). The other downregulated genes included previously known AR repressed genes, such as DDC, OPRK1, NOV and SERPIN1 (Fig. 3A, 3B). We then examined the role of AR signaling in the regulation of TRIM59 using PCa cell lines. As shown in Fig. 3C, stimulation of LNCaP cells with synthetic androgen R1881 (2nM) led to an activation of AR signaling evidenced by the upregulation of AR target genes $K L K 3$ and NKX3.1. AR activation resulted in a significant decrease in the expression of TRIM59 both at mRNA and protein levels (Fig. 3C). Treatment with the ARPI, Enzalutamide (20 MM), remarkably increased the TRIM59 transcription and protein levels, accompanied with reduced expression of androgen-driven genes KLK3 and NKX3.1 (Fig. 3C). Moreover, we knocked down or overexpressed AR in LNCaP and LAPC4 cells and examined changes in TRIM59 expression. Consistent with the AR antagonist and agonist treatment experiment results, LNCaP and LAPC4 cells exhibited a significant increase in the expression of TRIM59 after AR knockdown, and a robust decrease in the TRIM59 expression when AR was overexpressed (Fig. 3D-F). To test whether the elevated TRIM59 expression by AR signaling blockage was a transient phenomenon, LNCaP cells were treated with 10 $\mu \mathrm{M}$ enzalutamide for 3 months to establish a long-term enzalutamide-treated LNCaP cell line (Fig. 3G). We observed a persistent higher expression of TRIM59 in the enzalutamide-resistant LNCaP cells compared to control cells (Fig. 3H, 3l).

To explore the mechanism underlying the negative regulation of TRIM59 by AR signaling, we cloned the promoter region of TRIM59 (-1462bp $\sim+1032 \mathrm{bp}$ ) and utilized a promoter driven luciferase assay to examine the impact of AR signaling on transcription of TRIM59. As shown in Fig. 3J, a significant decrease in the luciferase activity was observed in R1881-stimulated LNCaP cells. We then analyzed the promoter region of TRIM59 in search of transcriptional factor binding sites using JASPAR, an online transcription factor binding profile database. Interestingly, 3 putative androgen response elements (AREs) which were located in -861bp -845bp, -589bp -573bp, and + 550bp +566bp were identified in the TRIM59 promoter (Fig. 3K). To validate those predicted ARE sites, we performed ChIP-qPCR for AR on LNCaP cells treated with $2 \mathrm{nM}$

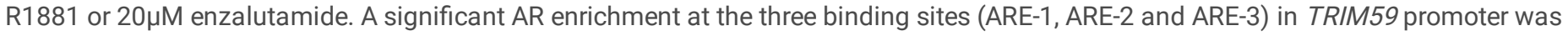
observed in R1881-stimulated LNCaP cells with respect to DMSO control (Fig. 3L). AR recruitment at the three AREs was reversed in the presence of enzalutamide (Fig. 3L). To further investigate how the transcription of TRIM59 was repressed by AR, we performed ChIP-qPCR for gene-expression-related epigenetic markers including H3K4me3, H3K27ac and H3K9me3. Among all the epigenetic modifications we examined, the most differentially changed epigenetic marker along with AR activation or suppression on TRIM59 promoter AREs was H3K9me3, a repressive histone modification (supplementary Fig. 3A). Additionally, knockdown of EHMT1/EHMT2, histone methyltransferases to specifically methylate $\mathrm{H} 3 \mathrm{~K} 9$, resulted in a significant upregulation in TRIM59 transcription (supplementary Fig. 3B). Together these findings 
support the notion that AR functioned as a direct transcriptional repressor of TRIM59. Attenuation of the AR signaling via potent ARPIs, such as enzalutamide, relieved the transcriptional repression of TRIM59 by AR.

\section{TRIM59 significantly promotes development of castration resistance and NED in PCas}

We then examined the functional significance of TRIM59 in PCas. As shown in supplementary Fig. 4, overexpression of TRIM59 in hormonaltherapy sensitive PCa cell lines LNCaP and LAPC4 resulted in a significant increase in tumor cell proliferation, migration and sphere forming capacity in vitro and an accelerated xenograft tumor growth in vivo. Importantly, we observed development of castration resistance in TRIM59-overexpressing LNCaP or LAPC4 cell lines, manifested as an enhanced cell proliferation rate and sphere forming capacity in ARPIcontaining medium compared to control cells (Fig. 4A-C). To further test the role of TRIM59 in the development of castration resistance of PCas in vivo, we implanted TRIM59-overexpressing or control LAPC4 cells subcutaneously to the castrated nude mice (Fig. 4D). Consistent with the in vitro experimental results, a notably castration independent xenograft growth was found in the TRIM59-overexpressing group, which stood in great contrast to the rare forming of xenograft tumor in control cell implanted group (Fig. 4E). Immunofluorescent staining of LAPC4 xenografts revealed prominent expression of neuroendocrine markers (SYP, NCAM-1 and CgA) in the TRIM59-overexpressing group but not in the control group (Fig. 4F). Knockdown of TRIM59 in CRPC cell lines PC3 and DU145, on the other hand, significantly inhibited the proliferation, sphere formation, migration in vitro and in vivo growth of tumor cells (Fig. 5). These data together suggested that TRIM59 functioned as an important factor in promoting castration resistance and NED in PCa.

We next conducted RNA-sequencing to compare the gene expression profile between LNCaP-TRIM59 and LNCaP control cells. In compliance with the previous results, TRIM59 overexpression resulted in increased transcription of neuroendocrine marker SYP, ENO2, and AURKA, along with a dramatic decline in AR signaling signature genes (Fig. 6A). Those gene expression alterations were further validated at protein levels by immunoblotting (Fig. 6B). Moreover, Gene Set Enrichment Analysis (GSEA) showed that luminal signature genes were markedly downregulated, while the small cell lung cancer (SCLC) gene set were significantly enriched in the LNCaP-TRIM59 cell line (Fig. 6C, 6D). Nervous system development related genes were preferentially expressed in LNCaP-TRIM59 revealed by GO pathway enrichment analysis (Fig. 6E, 6F). Interestingly, we found that AR signaling signature genes were significantly downregulated in TRIM59 overexpressing LNCaP cells, which was revealed both by q-PCR and GSEA analysis of RNA-seq data (supplementary Fig. 5), suggesting a negative feedback between TRIM59 and AR signaling in PCa cells. To determine whether TRIM59 was necessary in the NED process, we next constructed TRIM59 knockdown in LNCaP cells and subjected the LNCaP-shTRIM59 or control cells to ARPI-containing medium. shRNA-mediated silencing of TRIM59 prevented the enzalutamide-induced upregulation of NE markers in LNCaP cells (Fig. 6G, 6H). Together these findings supported an essential role of TRIM59 in PCa cellular plasticity and NED.

\section{TRIM59 drives the NED of prostate cancer by promoting degradation of RB1 and P53}

To further elucidate the mechanism of TRIM59 in NED, we carefully analyzed the expression alterations of previously reported neuroendocrine driver genes in LNCaP-TRIM59 RNA-seq data (Fig. 6A). Among them, the lineage plasticity gene SOX2 was the mostly differentially expressed one in LNCaP-TRIM59 cells. Interestingly, GSEA analysis on the LNCaP-TRIM59 RNA-seq data showed that the RB1 and P53 target geneset were significantly underexpressed in LNCaP-TRIM59 cells (Fig. 7A), while SOX2 downstream targets were markedly enriched (Fig. 7B). It has been demonstrated that concurrent loss or mutation of RB1 and TP53 were the most common genetic alterations in NEPC[9]. SOX2 was found as a downstream pivotal factor of RB1 and TP53 loss in promoting lineage plasticity and NED [12]. In agreement with our GSEA analysis, we found declines in both RB1 and P53 protein levels in LNCaP and LAPC4 cell lines overexpressing TRIM59 (Fig. 7C). Meanwhile the RB1 and P53 downregulation was accompanied with a rising level of the SOX2 protein. We did not detect overt changes in RB1 and TP53 mRNA levels upon TRIM59 overexpression, suggesting that the downregulation of RB1 and P53 was at post-translational level (Fig. 7D). We then investigated the impact of ectopic expression of TRIM59 on RB1 and P53 protein stability. As shown in Fig. 7E, TRIM59 overexpression led to an accelerated downregulation of protein abundance of RB1 and P53 in 293T cells. Similarly, we found that TRIM59 promoted reduction of endogenous RB1 and P53 levels in LNCaP cells in a time-dependent and dose-dependent manner (Fig. 7F, 7G). We further performed a time-dependent protein stability assay with the addition of $10 \mu \mathrm{g} / \mathrm{ml}$ protein synthesis inhibitor $\mathrm{CHX}$ with or without $10 \mu \mathrm{M}$ proteasome inhibitor MG132. As shown in Fig. 7H, TRIM59 significantly reduced the half-life of RB1 and P53 proteins, which could be reversed by addition of MG132. These data suggested that TRIM59 promoted RB1 and P53 degradation via the proteasome pathway.

To further analyze whether TRIM59 induced NED was acted via downregulating RB1 and P53, we used two cell line models with NED phenotype, that were LNCaP-ENZA-R cells (Fig. 3G) and LNCaP cells stably transfected with RB1 and TP53 shRNA as previously reported[11]. As shown in Fig. 7l, we detected a prominent decline of neuroendocrine markers NSE and SYP, as well as SOX2 in LNCaP-ENZA-R cells upon TRIM59 silencing. In contrast, no significant alterations in expression levels of NSE, SYP and SOX2 was found in a shRB1/sh TP53 stably 
transfected LNCaP cells after TRIM59 silencing (Fig. 7J). These data suggested that the neuroendocrine phenotype induced by TRIM59 is dependent on RB1 and P53. To further test the role of SOX2 in TRIM59-induced PCa NED, we conducted shRNA-mediated SOX2 silencing in LNCaP-TRIM59 cells. Importantly, we found that SOX2 knockdown led to a significant reduction of NE markers (NCAM-1, SYP, CgA) in LNCaPTRIM59 cells (Fig. 7K). Therefore, TRIM59 was an AR-suppressed key molecular driver for lineage plasticity and neuroendocrine differentiation in prostate cancer, which acted through enhanced RB1/P53 degradation and upregulation of SOX2 (Fig. 8).

\section{Discussion}

In the current study, analysis from published DNA and RNA sequencing data of prostate adenocarcinomas and NEPCs, together with IHC staining of tumor tissues from our large in-house CRPC cohort consistently show a marked elevation of TRIM59 expression in t-NEPC. We further demonstrate that upregulation of TRIM59 promotes the lineage transdifferention of PCa cells with a luminal phenotype to the t-NEPC phenotype characterized with underexpression of luminal genes and increased transcription of neuroendocrine maker genes. Notably, this phenotype change is associated with enhanced resistance to the APRI treatment in vitro and in vivo. TRIM59 knockdown significantly abrogates NED in PCa cells treated with ARPIs. Altogether, we uncover that TRIM59, an oncogenic TRIM family protein, as an essential molecular driver for the highly lethal t-NEPC formation.

Except for a small proportion of de-novo NEPC, most of NEPCs are induced by potent ARPI therapies. Tracking the change of gene expression characteristics or DNA mutations of the PCa biopsy samples in the same patient before and after ARPI treatment can provide valuable information to elucidate the molecular basis of t-NEPC progression. However, the samples from the same patient are often difficult to obtain. We have carefully collected 10 pairs of before-and-after samples from prostate adenocarcinoma patients who received ARPI therapy and eventually develop t-NEPC in the past 10 years at Ren Ji Hospital, one of the largest PCa treatment center in China. IHC staining of these sequential samples provides compelling evidence that TRIM59 upregulation is frequently found in ARPI resistant NEPC samples compared to hormone naïve PCa at first diagnosis. Those data strongly support that the expression of the NED driver gene TRIM59 is induced upon ARPI treatment.

Reports over past few years have highlighted how ARPI treatment [3] or loss of AR signaling promotes the NED of PCa cells. A few genes that have been reported to associate with an NEPC phenotype, such as BRN2[14] are controlled by AR. Yet how AR activity loss modulates the tNEPC-inducing molecular events is not fully understood. Our current study demonstrates that AR directly binds to TRIM59 promoter and represses its transcription. ARPI treatment leads to a reversal of the suppressive histone modifications on TRIM59 gene and a release of transcriptional restraint on TRIM59 by AR. We further uncover that the repressive epigenetic modification H3K9me3 in the TRIM59 promoter is dynamically changed along with AR activation or suppression. Knockdown of H3K9me3 methyltransferases releases the transcription repression of TRIM59. Therefore, an androgen-dependent epigenetic program contributes to the regulation of TRIM59 transcription. In addition, we detect a negative feedback between TRIM59 and AR signaling in PCa cells, based on the observation that enforced expression of TRIM59 leads to a suppression of AR target genes. The present work provides insights into the reciprocal negative regulation between AR signaling and TRIM59 in NED.

The concurrent loss or mutations of $R B 1$ and TP53 is reported to be the most frequent genetic alterations in NEPC [3, 9, 11]. Mechanistic study has shown that RB1 and TP53 loss mediated NED of PCa is dependent on the reprograming transcriptional factor SOX2[12]. In addition, an independent group also reported that RB1 protein loss was found in $90 \%$ of NEPC cases, whereas $23 \%$ cases harbored homozygous loss of $R B 1[10]$. This observation suggests that alternative mechanisms are taken to downregulate RB1 and P53 in NEPCs carrying no RB1/TP53 genetic alterations. In this study, we find that TRIM59 promotes the degradation of RB1 and P53 in PCa cells. Moreover, the NED-inducing effect of TRIM59 overexpression is dependent on RB1 and P53. We further demonstrate that knockdown of the reprograming factor SOX2 significantly overrides the NED phenotype in TRIM59 overexpressed PCa cells. Therefore, TRIM59 drives the NED of prostate cancer by enhancing the degradation of P53 and RB1 and subsequently upregulating downstream lineage plasticity promoting transcription factor SOX2. This provides an explanation of downregulation in RB1 and P53 proteins in NEPCs without RB1/TP53 genetic alterations

The emergence of t-NEPC is a complicated pathological process. Understanding how AR activity loss and key molecular drivers trigger this pathological lineage transdifferentiation process is essential to overcome resistance of current ARPI therapies and to develop novel treatment approaches. Our data from human PCa patient sample examinations, in vitro and in vivo functional analysis and mechanistic study collectively demonstrate that TRIM59 is an AR suppressed oncogenic protein and acts as an essential driver in the progression treatmentinduced NEPC.

\section{Conclusion}

In summary, we identify TRIM59 as an AR-suppressed molecular driver of neuroendocrine differentiation in prostate cancer. Mechanistically, ARPI treatment leads to a reversal of transcriptional restraint on TRIM59 by AR. Upregulated TRIM59 then drives the NED of PCa by 
enhancing the degradation of RB1 and P53 and upregulating downstream lineage plasticity-promoting transcription factor SOX2. Our study provides a novel prognostic marker for PCas and shed new light on the molecular pathogenesis of t-NEPC, a deadly variant of PCa.

\section{Abbreviations}

PCa: prostate cancer

CRPC: castration resistant prostate cancer

CRPC-AD: CRPC with adenocarcinoma phenotype

NEPC: neuroendocrine prostate cancer

t-NEPC: treatment-induced neuroendocrine prostate cancer

NED: neuroendocrine differentiation

SCLC: small cell lung cancer

AR: androgen receptor

ARPI: androgen receptor pathway inhibitor

ARE: androgen response element

TCGA: The Cancer Genome Atlas

GSEA: gene set enrichment analysis

$\mathrm{CHX}$ : Cycloheximide

\section{Declarations}

\section{Ethics approval and consent to participate}

All animal experiments were approved by the Institutional Animal Care and Use Committee of Renji Hospital, School of Medicine, Shanghai Jiao Tong University.

\section{Consent for publication}

All subjects have written informed consent.

\section{Availability of data and material}

The RNA-sequencing datasets are available upon request.

\section{Competing interests}

The authors declare that they have no competing interests.

\section{Funding}

The study was supported by funds to H.H. Zhu from the National Key R\&D Program of China (2017YFA0102900), the National Natural Science Foundation of China (NSFC32022021, NSFC81772743 and NSFC81972404), the Shanghai Municipal Education CommissionGaofeng Clinical Medicine Grant Support (20181706), and the Innovative research team of high-level local universities in Shanghai. The study was also supported by funds to Department of Urology, Ren Ji hospital from National Natural Science Foundation of China (82002710, 81772742, 81672850), Shanghai Municipal Education Commission-Gaofeng Clinical Medicine Grant Support (20191906); Shanghai Sailing Program(20YF1425300).

\section{Author contributions}


W.X., H.H.Z., B.D., L.F. and Y.G. designed the study and wrote the manuscript; L.F. and Y.G. performed all the experiments and data analysis with the help from Y.H., Q.W. and W.G.; W.X. and B.D. provided clinical samples and patient information; all authors read and approved the final manuscript.

\section{Acknowledgements}

Not applicable.

\section{References}

1. de Bono JS, Logothetis CJ, Molina A, Fizazi K, North S, Chu L, Chi KN, Jones RJ, Goodman OB Jr, Saad F, et al. Abiraterone and increased survival in metastatic prostate cancer. N Engl J Med. 2011;364:1995-2005.

2. Scher HI, Fizazi K, Saad F, Taplin ME, Sternberg CN, Miller K, de Wit R, Mulders P, Chi KN, Shore ND, et al. Increased survival with enzalutamide in prostate cancer after chemotherapy. N Engl J Med. 2012;367:1187-97.

3. Aggarwal R, Huang J, Alumkal JJ, Zhang L, Feng FY, Thomas GV, Weinstein AS, FriedI V, Zhang C, Witte ON, et al. Clinical and Genomic Characterization of Treatment-Emergent Small-Cell Neuroendocrine Prostate Cancer: A Multi-institutional Prospective Study. J Clin Oncol. 2018;36:2492-503.

4. Aparicio AM, Harzstark AL, Corn PG, Wen S, Araujo JC, Tu S-M, Pagliaro LC, Kim J, Millikan RE, Ryan C, et al: Platinum-Based Chemotherapy for Variant Castrate-Resistant Prostate Cancer. 2013, 19:3621-3630.

5. Wang ZA, Shen MM. Revisiting the concept of cancer stem cells in prostate cancer. Oncogene. 2011;30:1261-71.

6. Wang ZA, Mitrofanova A, Bergren SK, Abate-Shen C, Cardiff RD, Califano A, Shen MM. Lineage analysis of basal epithelial cells reveals their unexpected plasticity and supports a cell-of-origin model for prostate cancer heterogeneity. Nat Cell Biol. 2013;15:274-83.

7. Quintanal-Villalonga Á, Chan JM, Yu HA, Pe'er D, Sawyers CL, Sen T, Rudin CM. Lineage plasticity in cancer: a shared pathway of therapeutic resistance. Nat Rev Clin Oncol. 2020;17:360-71.

8. Davies AH, Beltran H, Zoubeidi A. Cellular plasticity and the neuroendocrine phenotype in prostate cancer. Nat Rev Urol. 2018;15:271-86.

9. Beltran H, Prandi D, Mosquera JM, Benelli M, Puca L, Cyrta J, Marotz C, Giannopoulou E, Chakravarthi BV, Varambally S, et al. Divergent clonal evolution of castration-resistant neuroendocrine prostate cancer. Nat Med. 2016;22:298-305.

10. Tan HL, Sood A, Rahimi HA, Wang W, Gupta N, Hicks J, Mosier S, Gocke CD, Epstein JI, Netto GJ, et al. Rb loss is characteristic of prostatic small cell neuroendocrine carcinoma. Clin Cancer Res. 2014;20:890-903.

11. Ku SY, Rosario S, Wang Y, Mu P, Seshadri M, Goodrich ZW, Goodrich MM, Labbe DP, Gomez EC, Wang J, et al. Rb1 and Trp53 cooperate to suppress prostate cancer lineage plasticity, metastasis, and antiandrogen resistance. Science. 2017;355:78-83.

12. Mu P, Zhang Z, Benelli M, Karthaus WR, Hoover E, Chen CC, Wongvipat J, Ku SY, Gao D, Cao Z, et al. SOX2 promotes lineage plasticity and antiandrogen resistance in TP53- and RB1-deficient prostate cancer. Science. 2017;355:84-8.

13. Zou M, Toivanen R, Mitrofanova A, Floch N, Hayati S, Sun Y, Le Magnen C, Chester D, Mostaghel EA, Califano A, et al. Transdifferentiation as a Mechanism of Treatment Resistance in a Mouse Model of Castration-Resistant Prostate Cancer. Cancer Discov. 2017;7:736-49.

14. Bishop JL, Thaper D, Vahid S, Davies A, Ketola K, Kuruma H, Jama R, Nip KM, Angeles A, Johnson F, et al. The Master Neural Transcription Factor BRN2 Is an Androgen Receptor-Suppressed Driver of Neuroendocrine Differentiation in Prostate Cancer. Cancer Discov. 2017;7:54-71.

15. Beltran H, Rickman DS, Park K, Chae SS, Sboner A, MacDonald TY, Wang Y, Sheikh KL, Terry S, Tagawa ST, et al. Molecular characterization of neuroendocrine prostate cancer and identification of new drug targets. Cancer Discov. 2011;1:487-95.

16. MYCN Transforms Prostate. Epithelium to Neuroendocrine Prostate Cancer. Cancer Discov. 2016;6:0f19.

17. Berger A, Brady NJ, Bareja R, Robinson B, Conteduca V, Augello MA, Puca L, Ahmed A, Dardenne E, Lu X, et al. N-Myc-mediated epigenetic reprogramming drives lineage plasticity in advanced prostate cancer. J Clin Invest. 2019;130:3924-40.

18. Dardenne E, Beltran H, Benelli M, Gayvert K, Berger A, Puca L, Cyrta J, Sboner A, Noorzad Z, MacDonald T, et al. N-Myc Induces an EZH2Mediated Transcriptional Program Driving Neuroendocrine Prostate Cancer. Cancer Cell. 2016;30:563-77.

19. Lee JK, Phillips JW, Smith BA, Park JW, Stoyanova T, McCaffrey EF, Baertsch R, Sokolov A, Meyerowitz JG, Mathis C, et al. N-Myc Drives Neuroendocrine Prostate Cancer Initiated from Human Prostate Epithelial Cells. Cancer Cell. 2016;29:536-47.

20. Yin Y, Xu L, Chang Y, Zeng T, Chen X, Wang A, Groth J, Foo WC, Liang C, Hu H, Huang J. N-Myc promotes therapeutic resistance development of neuroendocrine prostate cancer by differentially regulating miR-421/ATM pathway. Mol Cancer. 2019;18:11.

21. Li Y, Donmez N, Sahinalp C, Xie N, Wang Y, Xue H, Mo F, Beltran H, Gleave M, Wang Y, et al. SRRM4 Drives Neuroendocrine Transdifferentiation of Prostate Adenocarcinoma Under Androgen Receptor Pathway Inhibition. Eur Urol. 2017;71:68-78. 
22. Ozato K, Shin DM, Chang TH, Morse HC. 3rd: TRIM family proteins and their emerging roles in innate immunity. Nat Rev Immunol. 2008;8:849-60.

23. Tomar D, Singh R. TRIM family proteins: emerging class of RING E3 ligases as regulator of NF-kappaB pathway. Biol Cell. 2015;107:2240.

24. Zhou Z, Ji Z, Wang Y, Li J, Cao H, Zhu HH, Gao WQ. TRIM59 is up-regulated in gastric tumors, promoting ubiquitination and degradation of p53. Gastroenterology. 2014;147:1043-54.

25. Valiyeva F, Jiang F, Elmaadawi A, Moussa M, Yee SP, Raptis L, Izawa JI, Yang BB, Greenberg NM, Wang F, Xuan JW. Characterization of the oncogenic activity of the novel TRIM59 gene in mouse cancer models. Mol Cancer Ther. 2011;10:1229-40.

26. Sheng Y, Ji Z, Zhao H, Wang J, Cheng C, Xu W, Wang X, He Y, Liu K, Li L, et al. Downregulation of the histone methyltransferase SETD2 promotes imatinib resistance in chronic myeloid leukaemia cells. Cell Prolif. 2019;52:e12611.

27. Chu M, Chang Y, Li P, Guo Y, Zhang K, Gao W. Androgen receptor is negatively correlated with the methylation-mediated transcriptional repression of miR-375 in human prostate cancer cells. Oncol Rep. 2014;31:34-40.

28. Sang Y, Li Y, Song L, Alvarez AA, Zhang W, Lv D, Tang J, Liu F, Chang Z, Hatakeyama S, et al. TRIM59 Promotes Gliomagenesis by Inhibiting TC45 Dephosphorylation of STAT3. Cancer Res. 2018;78:1792-804.

29. Rudin CM, Poirier JT, Byers LA, Dive C, Dowlati A, George J, Heymach JV, Johnson JE, Lehman JM, MacPherson D, et al. Molecular subtypes of small cell lung cancer: a synthesis of human and mouse model data. Nat Rev Cancer. 2019;19:289-97.

30. Olsen JR, Azeem W, Hellem MR, Marvyin K, Hua Y, Qu Y, Li L, Lin B, Ke X, Øyan AM, Kalland K. Context dependent regulatory patterns of the androgen receptor and androgen receptor target genes. BMC Cancer. 2016;16:377.

\section{Figures}

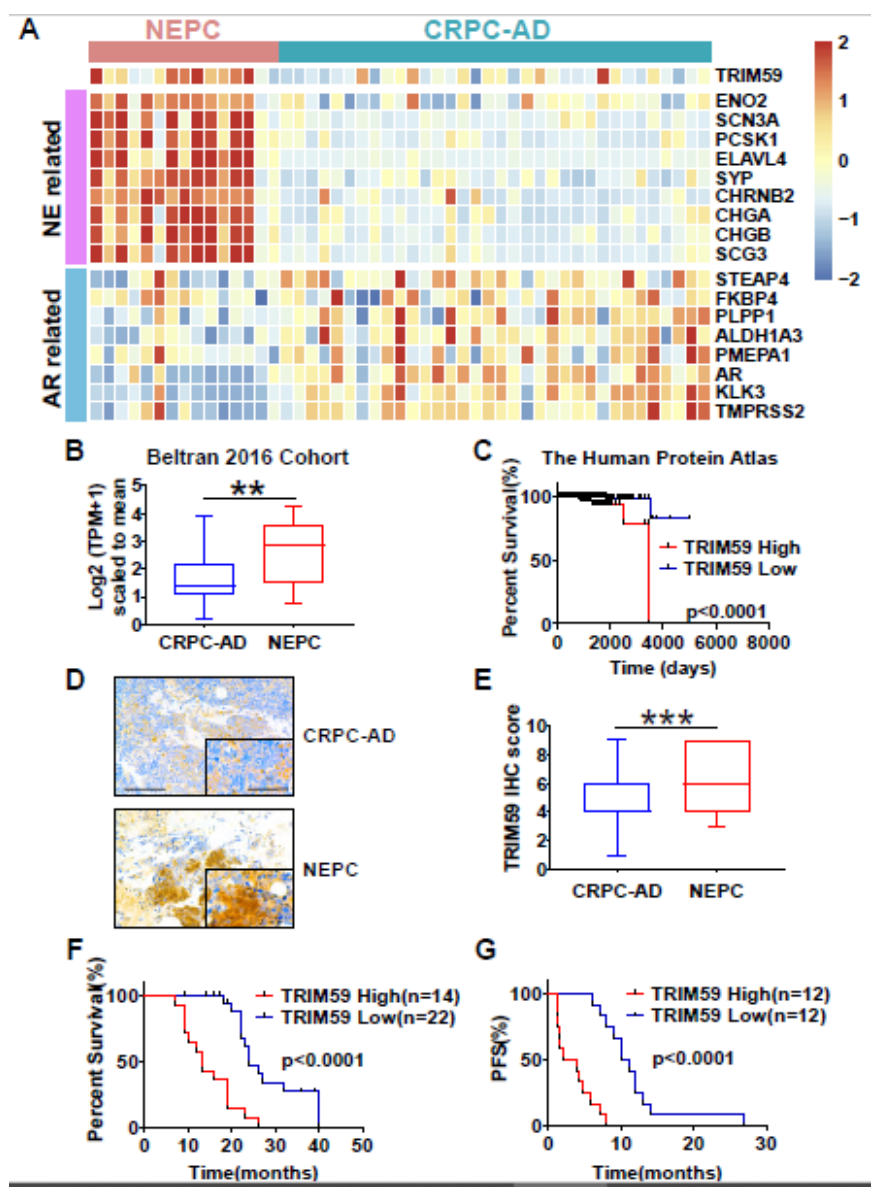

\section{Figure 1}

TRIM59 is highly expressed in castration resistant neuroendocrine prostate cancer and associated with poor disease outcome. (A) Heatmap depicting TRIM59 and selected genes expression in castration resistant neuroendocrine prostate cancer (NEPC, $\mathrm{n}=15)$ and castration resistant adenocarcinoma (CRPC-AD, $n=34)$ in the Beltran-2016 cohort[9] $(n=49$, expression values $=\log 2(T P M+1))$. (B) TRIM59 is upregulated in NEPC 
subgroup compared to CRPC-AD. (C) High expression level of TRIM59 is significantly associated with worse survival in PCa patients in The Human Protein Atlas database. (D) Representative images of IHC staining of TRIM59 in in our in-house CRPC cohort (RENJI-CRPC cohort) containing 39 CRPC-AD and 21 NEPC and CRPC-AD samples. Scale bars, left, $100 \mu \mathrm{m}$; right, $25 \mu \mathrm{m}$. (E) Quantification of TRIM59 staining intensity in RENJI-CRPC cohort. IHC score is calculated as the multiplied value of staining intensity index (0 3) and positive staining area index (0 3). (F) overall survival of patients stratified by TRIM59 expression in RENJI-CRPC cohort ( $n=36)$. (G) Progression free survival of patients who received abiraterone treatment in RENJI-CRPC cohort stratified by TRIM59 expression in RENJI-CRPC cohort ( $n=24)$. PFS represents progression free survival. (Two- tailed student's t test was applied in (B) and (E). Kaplan-Meier method and log-rank test are used to evaluate survival and compare the difference in $(C),(F)$ and $(G) .{ }^{*} p<0.01,{ }^{*} * * p<0.0001$.)

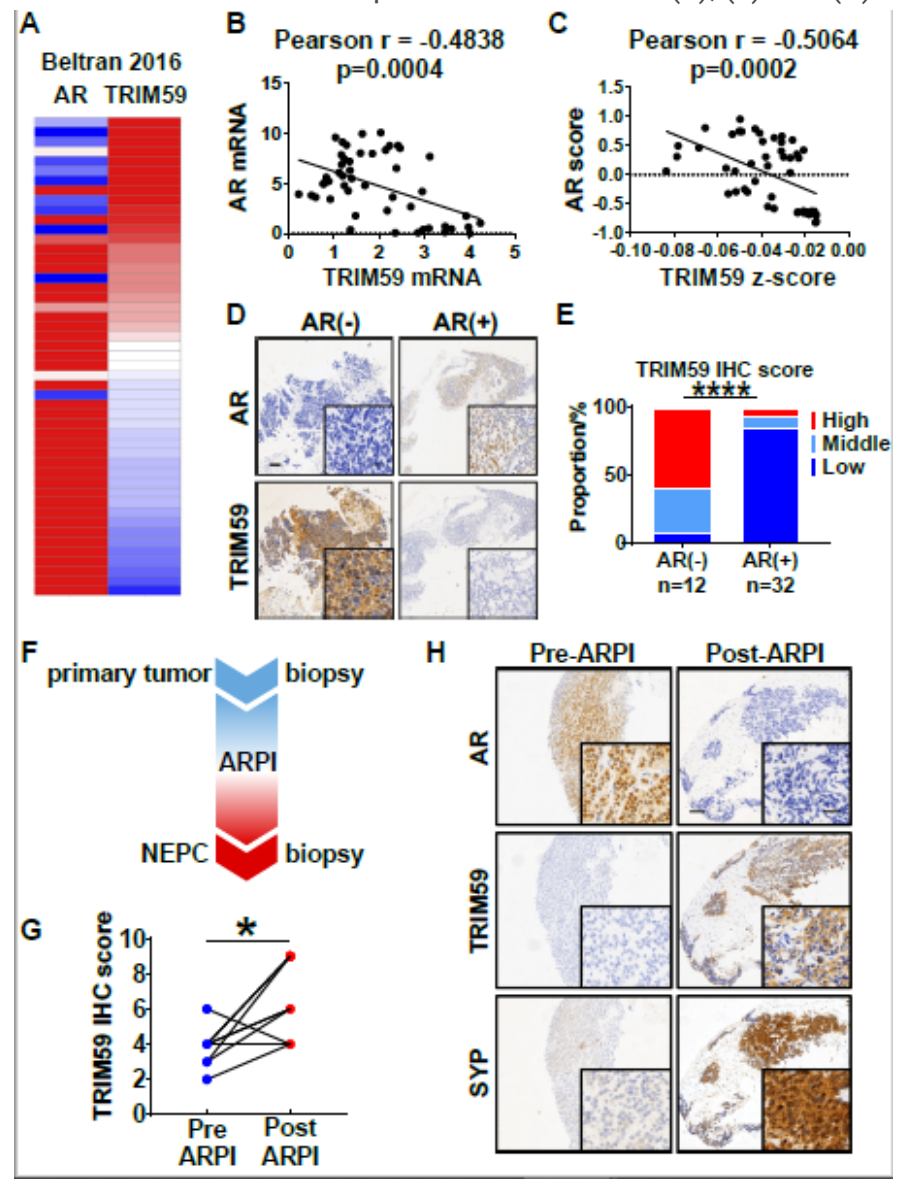

Figure 2

TRIM59 is negatively correlated with AR signaling in human prostate cancers (A) Heatmap depicting AR and TRIM59 mRNA levels in the Beltran-2016 cohort[9] ( $n=49)$. Shades of red and blue represent expression values in log2 (RPKM+1). (B) Pearson correlation analysis of TRIM59 mRNA and AR mRNA in the Beltran-2016 cohort $(n=49)$. (C) Pearson correlation analysis of TRIM59 z-score and AR score described in previous study in the Beltran-2016 cohort $(n=49)$. (D) IHC staining of AR and TRIM59 in the RENJI-CRPC cohort ( $n=44)($ Scale bar, left, $100 \mu \mathrm{m}$; right $20 \mu \mathrm{m})$. (E) Quantification of TRIM59 IHC staining score in the AR-negative (AR-) and AR-positive (AR+) prostate tumor samples from CRPC patients $(n=44)$. The Data is analyzed by rank sum test. ${ }^{\star \star *} p<<0.0001$. (F) Schematic diagram of sequential PCa sample collection from the same patient $(n=10)$ before and after ARPI treatment. (G) Quantification of TRIM59 IHC staining score in the paired sequential samples $(n=20)$ in $(f)$. paired-sample t test is used to compare the difference. * $p<0.05$. (H) IHC staining of AR, TRIM59 and SYP in paired PCa samples pre- and post-ARPI treatment (Scale bar=100 $\mu \mathrm{m}$, Left; Scale bar=20 $\mu \mathrm{m}$, Right). 


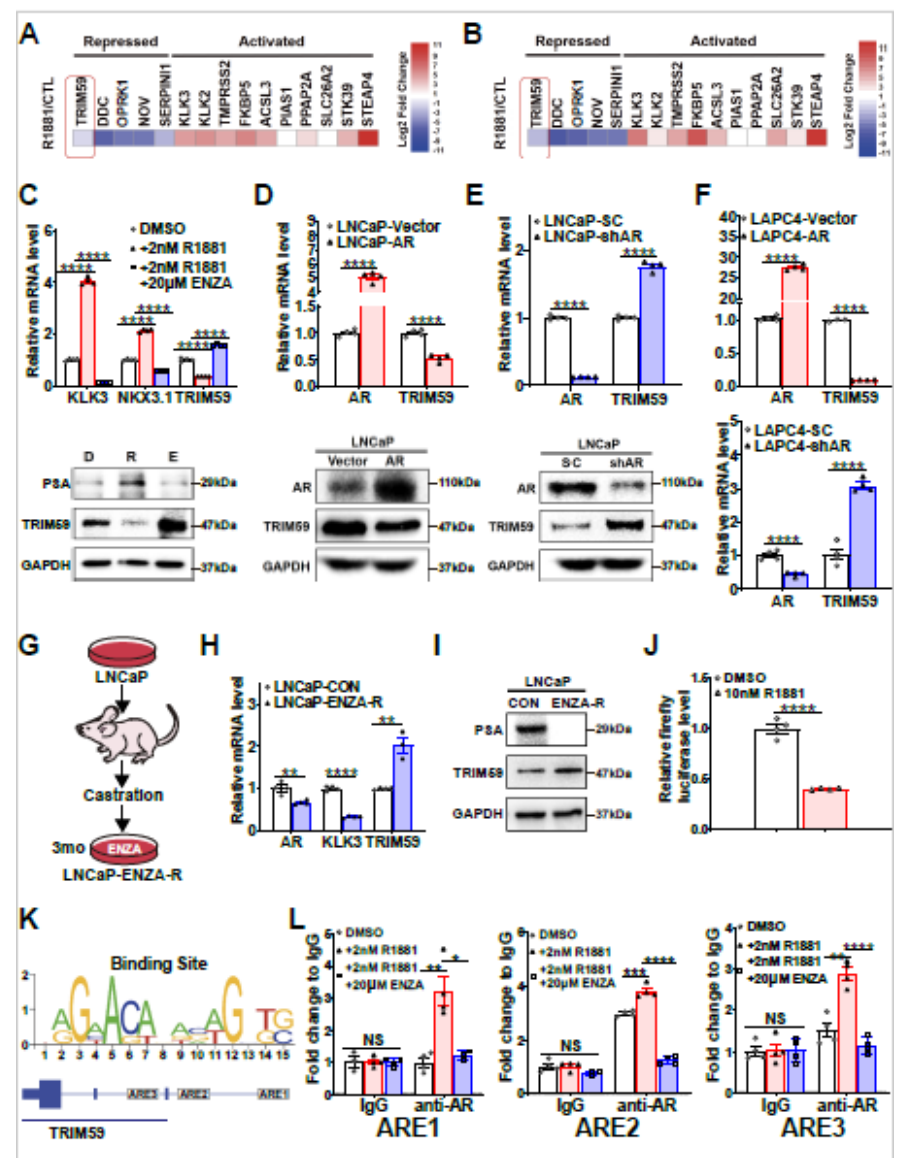

Figure 3

AR suppresses the transcription of TRIM59 in prostate cancer cells. (A, B) Heatmap depicting relative expression of androgen regulated genes in LNCaP (A) or VCaP cells (B) upon androgen (R1881) stimulation. Analysis is performed on published dataset (GSE71797)[30]. (C) q-PCR (upper panel) and immunoblots (lower panel) of indicated genes in LNCaP cells treated with R1881 with or without enzalutamide. GAPDH is used as the internal control. (D) q-PCR (upper panel) and immunoblots (lower panel) of AR and TRIM59 in stably AR overexpressing LNCaP cells and control cells. GAPDH is used as the internal control. (E) q-PCR (upper panel) and immunoblots (lower panel) of AR and TRIM59 in shAR and scramble shRNA transfected LNCaP cells. GAPDH is used as the internal control. (F) q-PCR analysis of TRIM59 and AR levels in stably AR overexpressing (upper panel) or shAR (lower panel) transfected LAPC4 cells. GAPDH is used as the internal control. (G) Schematic diagram of generation of enzalutamide-resistant LNCaP cells (LNCaP -ENZ-R). (H) q-PCR analysis of AR, KLK3 and TRIM59 in LNCaP- ENZA-R and LNCaP control cells. GAPDH is used as the internal control. (I) Immunoblots of PSA and TRIM59 in LNCaP- ENZA-R and LNCaP cells. GAPDH is used as the internal control. ( $J$ ) Schematic diagram represents AR binding motif (upper panel) and androgen response elements (ARE) (lower panel) located in the TRIM59 promoter. (K) Relative firefly luciferase activity in LNCaP transfected with pGL4.17-TRIM59promoter vector upon androgen stimulation. (L) ChIP-qPCR for AR on the TRIM59 promoter in LNCaP treated with R1881 or enzalutamide. (Means $\pm S E M$ in $(C),(D),(E),(F),(H),(K)$ and $(L)$. two-tailed unpaired Students $t$ test is used in $(C),(D),(E),(F),(H),(K)$ and $(L) .{ }^{*} p<0.05,{ }^{\star \star} p<$ $0.01, \star \star \star \mathrm{p}<0.001, \star \star \star \star \mathrm{p}<0.0001$.) 
A

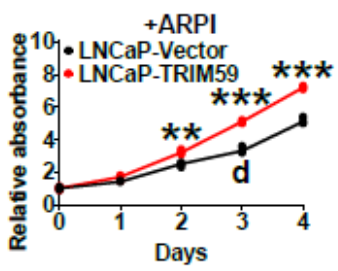

$c$

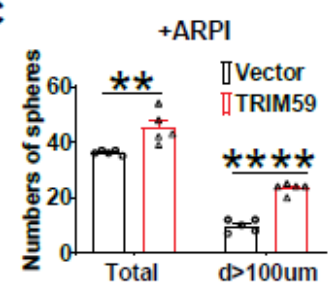

D

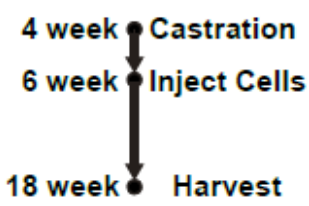

E

Vector

TRIM59

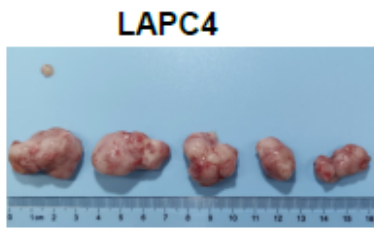

F
B
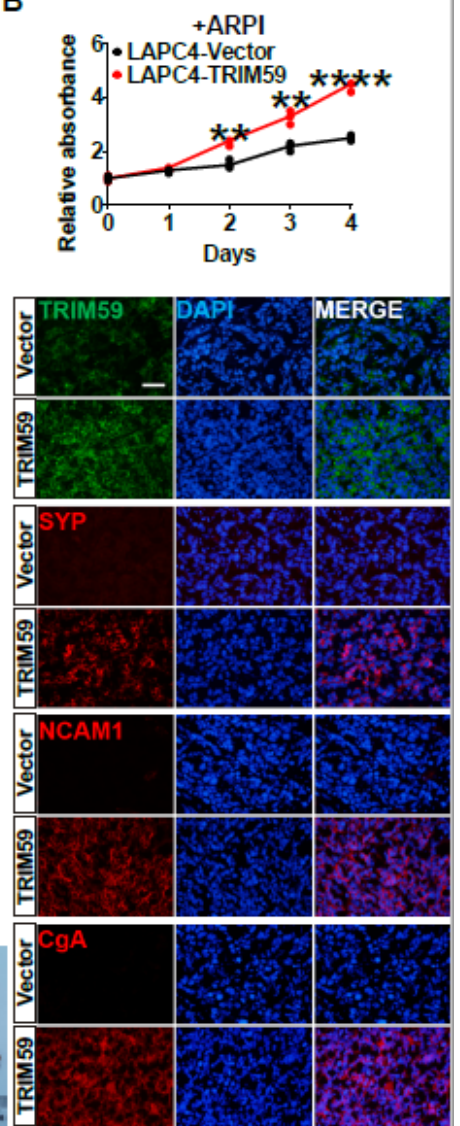

Figure 4

TRIM59 significantly promotes castration resistance in PCa cells. (A) Cell proliferation assay using stable TRIM59 expressing LNCaP and control cells cultured with ARPI ( $5 \mu \mathrm{m}$ bicalutamide). (B) Cell proliferation assay using stable TRIM59 expressing LAPC4 and control cells cultured with ARPI (5 $\mu \mathrm{m}$ bicalutamide). (C) Quantification of sphere numbers derived from LAPC-TRIM59 or control cells in normal or ARPI containing medium. (D) Schematic diagram of generation of subcutaneous xenografts using stable TRIM59 expressing LAPC4 and control cells. (E) Image showing LAPC4 xenografts $(n=5)$ in castrated nude mice harvested 12 weeks post implantation. ( $F)$ Immunofluorescence staining of TRIM59 and indicated NE markers in LAPC4 xenografts in castrated nude mice. (Means \pm SEM in (C). Two-tailed unpaired Students t test is used in (A), (B) and (C). * $p<0.05, * * p<0.01, * \star * p<0.001, * \star \star * p<0.0001$.) 
A

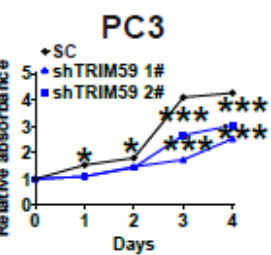

C

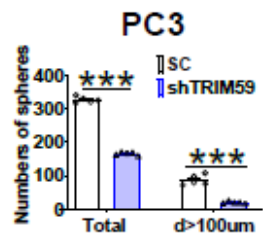

E

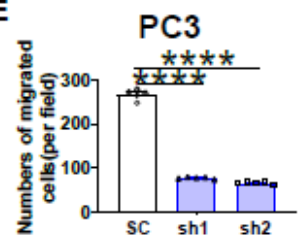

B

DU145

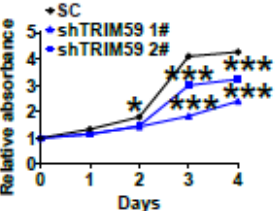

D

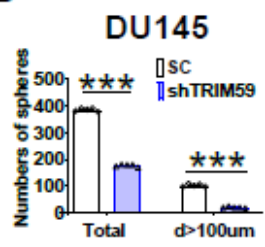

F

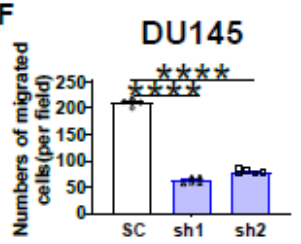

G
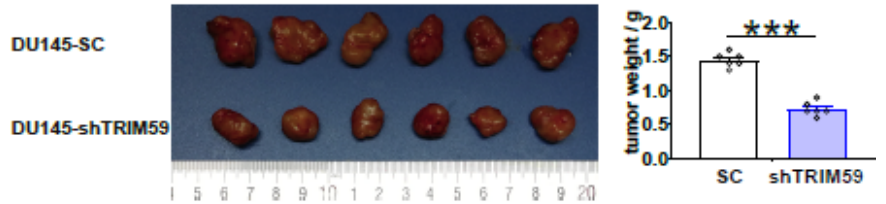

Figure 5

TRIM59 silencing suppress CRPC cell proliferation, migration and xenograft tumor growth. (A, B) Cell proliferation assay in shRNA-mediated TRIM59 silencing PC3 (A), DU145 (B) and respectively control cells ( $n=3)$. (C, D) Sphere formation assay in shRNA-mediated TRIM59 silencing PC3 (C), DU145 (D) and respectively control cells ( $n=5)(E, F)$ Cell migration assay in shRNA-mediated TRIM59 silencing PC3 (E), DU145 (F) and respectively control cells $(n=5)(G)$ Subcutaneous xenograft assay using shRNA-mediated TRIM59 silencing DU145 ( $n=6)$ and respectively control cells. Representative image (left panel) shows the tumor samples. Bar graph (right panel) shows the tumor weight in each

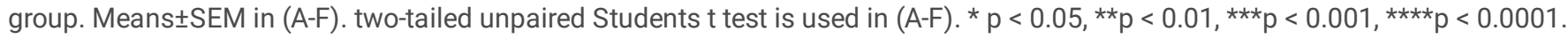




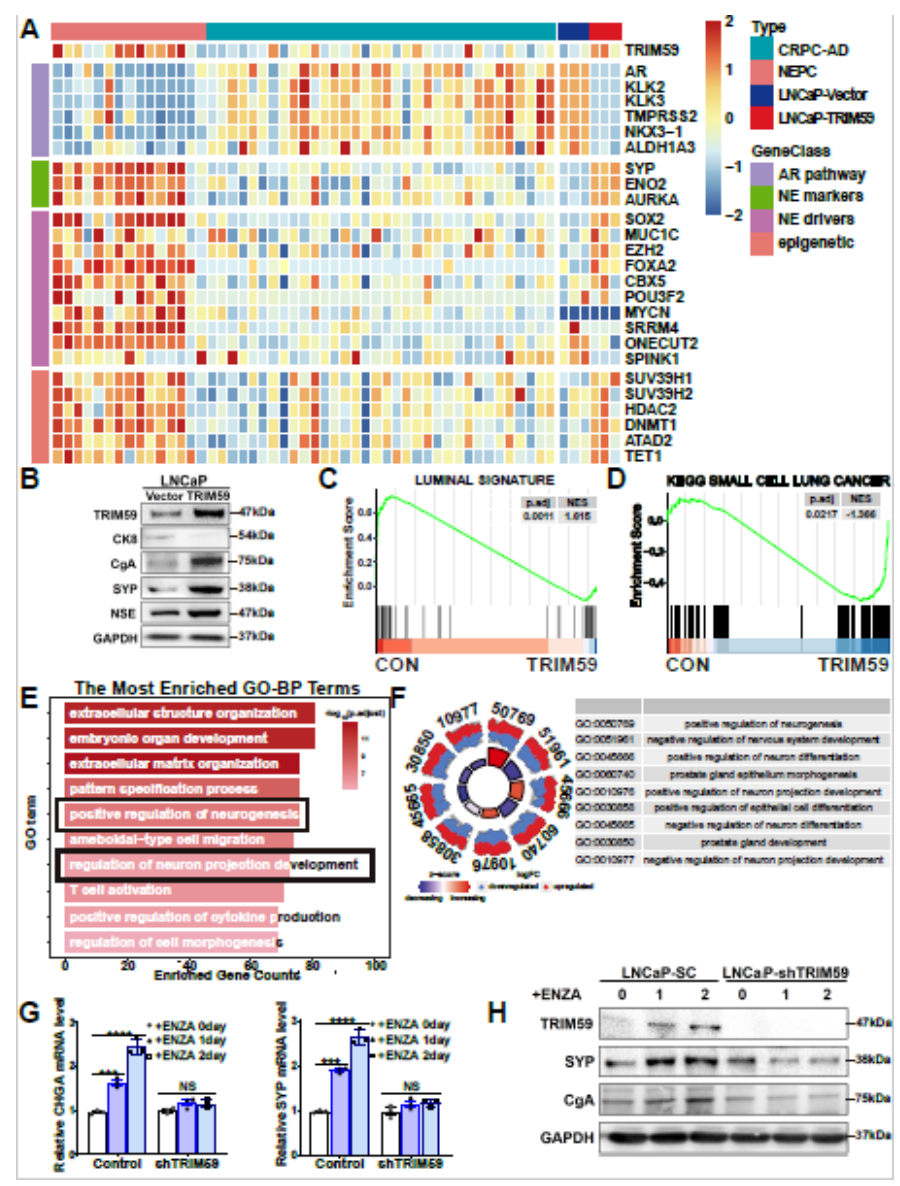

Figure 6

TRIM59 drives the NED of PCa. (A) Heatmap depicting TRIM59 and selected genes expression in stable TRIM59 expressing LNCa P ( $n=3)$ and control cells $(n=3)$ along with the Beltran-2016 cohort[9] $(n=49$, mRNA expression value= log2 (TPM+1)). (B) Immunoblots of TRIM59 and indicated NE markers in stable TRIM59 expressing LNCaP cells and control cells. GAPDH is used as a loading control. (C-D) Gene Set Enrichment Analysis of luminal cell gene signature (C) and small cell lung cancer signature (D). (E) the top 10 enriched processes in gene ontology (GO) enrichment analysis of RNA-Seq data from LNCaP-TRIM59 and LNCaP-vector control cells. The shades of color represent p value. The length of bar represents number of enriched genes. (F) gene ontology (GO) term enrichment analysis of selected pathway related to neuronal or prostate development in TRIM59 expressing LNCaP $(n=3)$ and control cells $(n=3)$. The shades of color represent $z$-score of each

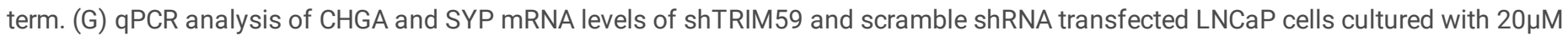
enzalutamide for indicated time. (H) Immunoblots of CgA, SYP and TRIM59 in samples described in (G). 


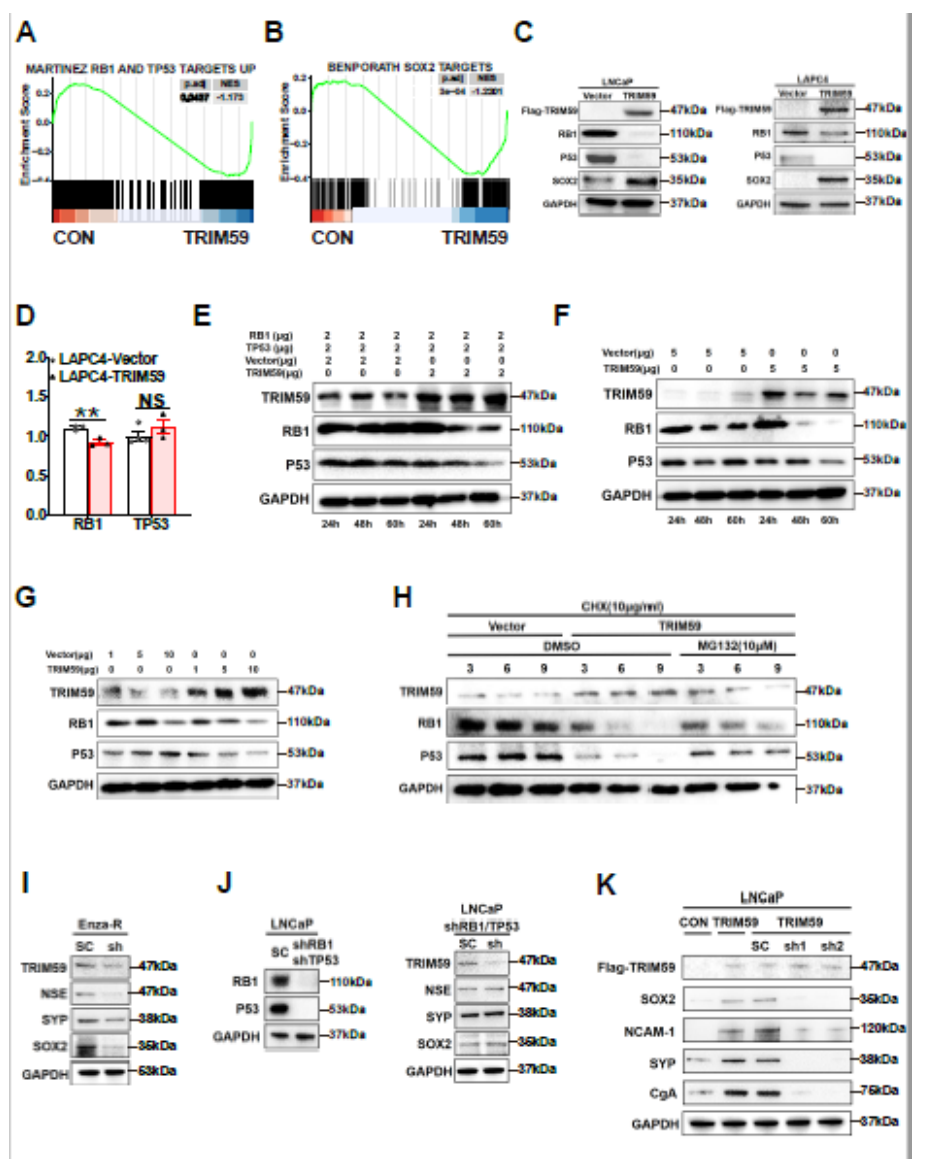

Figure 7

TRIM59 promotes degradation of P53 and RB1 and upregulates SOX2. (A) GSEA analysis of "MARTINEZ RB1 AND TP53 TARGETS UP" in stable TRIM59 expressing LNCaP $(n=3)$ and control cells $(n=3)$. (B) GSEA analysis of "BENPORATH SOX2 TARGET" in stable TRIM59 expressing LNCaP $(n=3)$ and control cells $(n=3)$. (C) Immunoblots of Rb, p53 and SOX2 in in stable TRIM59 expressing LNCaP or LAPC4 and respective control cells. GAPDH is used as a loading control. (D) q-PCR of Rb and p53 in stable TRIM59 expressing LNCaP and control cells. GAPDH is used as the internal control. (E) TRIM59 expression shortens the half-life of exogenous Rb and p53 protein levels in 293T cells cotransfected with 2ug P53-expressing and 2ug RB1-expressing plasmids. (F) TRIM59 expression shortens the half-life of endogenous Rb and p53 protein in LNCaP cells. (G) The protein levels of Rb and p53 decreases correspondingly with increasing TRIM59 level. (H) Addition of 10 $\mu$ M MG132 reverses TRIM59-mediated reduced half-life of endogenous Rb and p53 in LNCaP cells. (I) Immunoblots of RB1, P53 in doubleknockdown LNCaP cells (DKD) and control cells. (J) Immunoblots of TRIM59, NSE, SYP and SOX2 in enzalutamide-resistant LNCaP cells, double-knockdown LNCaP cells (DKD) and their shTRIM59 cells. (K) Immunoblots of Flag-TRIM59, SOX2 and indicated NE markers in SOX2silencing LNCaP-TRIM59 cells and control cells. 


\section{DHT}

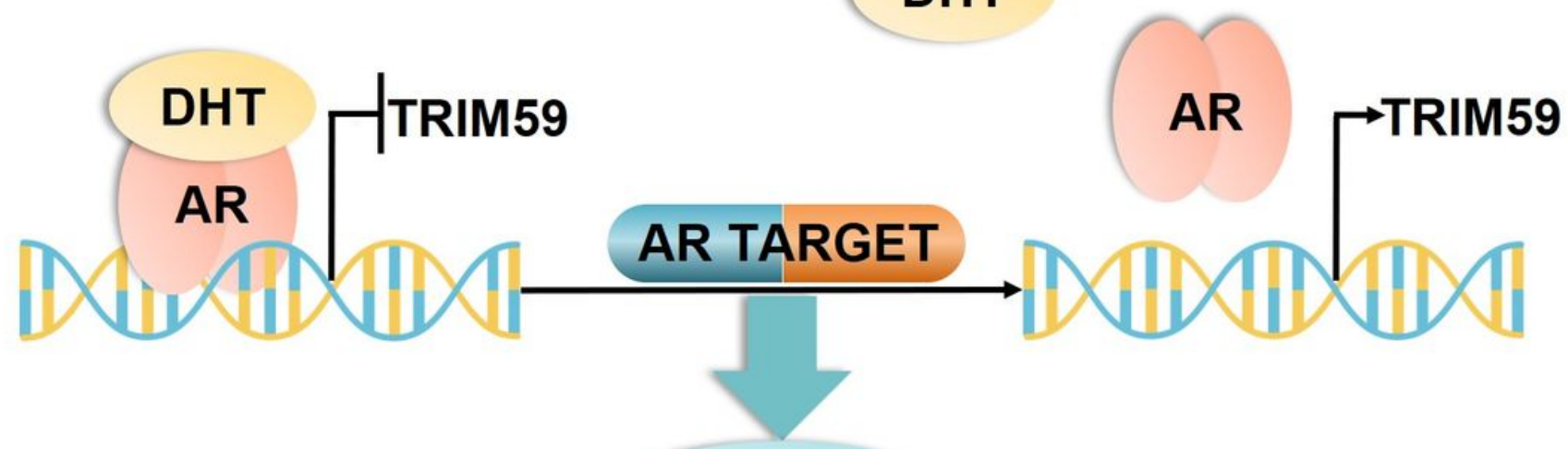

\section{Prostate
adenocarcinoma \\ Prostate
adenocarcinoma}
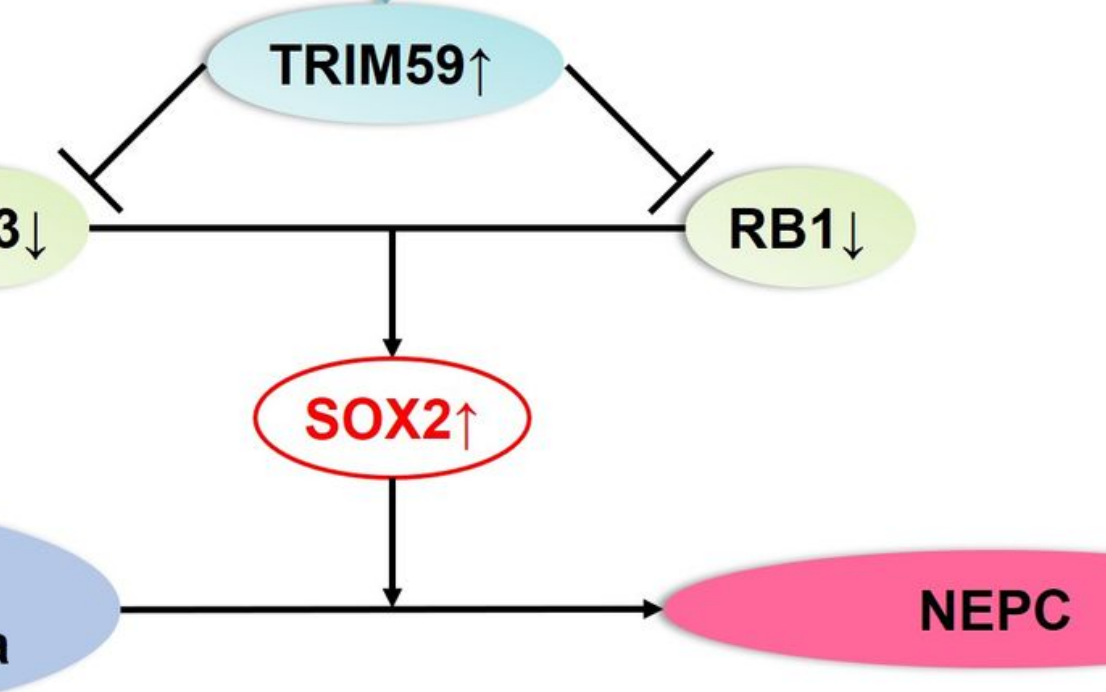

Figure 8

Figure 8

Schema illustrating the molecular mechanism of TRIM59 as an AR suppressed key driver to promote neuroendocrine differentiation in prostate cancer.

\section{Supplementary Files}

This is a list of supplementary files associated with this preprint. Click to download.

- TRIM59NEPCsupplementarymaterials.docx 